\title{
Solvability for Two Classes of Higher-Order Multi-Point Boundary Value Problems at Resonance
}

\author{
Yunzhu Gao and Minghe Pei \\ Department of Mathematics, Beihua University, Jilin City 132013, China
}

Correspondence should be addressed to Minghe Pei, peiminghe@ynu.ac.kr

Received 13 July 2007; Revised 14 December 2007; Accepted 29 January 2008

Recommended by Ivan Kiguradze

Using the theory of coincidence degree, we establish existence results of positive solutions for higher-order multi-point boundary value problems at resonance for ordinary differential equation $u^{(n)}(t)=f\left(t, u(t), u^{\prime}(t), \ldots, u^{(n-1)}(t)\right)+e(t), \quad t \in(0,1)$, with one of the following boundary conditions: $u^{(i)}(0)=0, i=1,2, \ldots, n-2, u^{(n-1)}(0)=u^{(n-1)}(\xi), u^{(n-2)}(1)=\sum_{j=1}^{m-2} \beta_{j} u^{(n-2)}\left(\eta_{j}\right)$, and $u^{(i)}(0)=0$, $i=1,2, \ldots, n-1, u^{(n-2)}(1)=\sum_{j=1}^{m-2} \beta_{j} u^{(n-2)}\left(\eta_{j}\right)$, where $f:[0,1] \times \mathbb{R}^{n} \rightarrow \mathbb{R}=(-\infty,+\infty)$ is a continuous function, $e(t) \in L^{1}[0,1] \beta_{j} \in \mathbb{R}(1 \leq j \leq m-2, m \geq 4), 0<\eta_{1}<\eta_{2}<\cdots<\eta_{m-2}<1,0<\xi<1$, all the $\beta_{-j}^{-s}$ have not the same sign. We also give some examples to demonstrate our results.

Copyright $(2008$ Y. Gao and M. Pei. This is an open access article distributed under the Creative Commons Attribution License, which permits unrestricted use, distribution, and reproduction in any medium, provided the original work is properly cited.

\section{Introduction}

In recent years, the multi-point boundary value problem (BVP) for second- or third-order ordinary differential equation has been extensively studied, and a series of better results is obtained in [1-10]. But the multi-point boundary value problems for higher order are seldom seen $[11,12]$.

In this paper, we consider the following higher-order differential equation:

$$
u^{(n)}(t)=f\left(t, u(t), u^{\prime}(t), \ldots, u^{(n-1)}(t)\right)+e(t), \quad t \in(0,1),
$$

with one of the following boundary conditions:

$$
\begin{gathered}
u^{(i)}(0)=0, \quad i=1,2, \ldots, n-2, \quad u^{(n-1)}(0)=u^{(n-1)}(\xi), \quad u^{(n-2)}(1)=\sum_{j=1}^{m-2} \beta_{j} u^{(n-2)}\left(\eta_{j}\right), \\
u^{(i)}(0)=0, \quad i=1,2, \ldots, n-1, \quad u^{(n-2)}(1)=\sum_{j=1}^{m-2} \beta_{j} u^{(n-2)}\left(\eta_{j}\right),
\end{gathered}
$$


where $f:[0,1] \times \mathbb{R}^{n} \rightarrow \mathbb{R}=(-\infty,+\infty)$ is a continuous function, $e(t) \in L^{1}[0,1], m \geq 4, n \geq 2$ are two integers, $\beta_{j} \in \mathbb{R}, \eta_{j} \in(0,1)(j=1,2, \ldots, m-2)$ are constants satisfying $0<\eta_{1}<\eta_{2}<\cdots<$ $\eta_{m-2}<1$.

For certain boundary condition case such that the linear operator $L u=u^{(n)}$, defined in a suitable Banach space, is invertible, this is the so-called nonresonance case, otherwise, the so-called resonance case $[2,9,10,12]$.

The purpose of this paper is to study the existence of solutions for BVP (1.1), (1.2) and BVP (1.1), (1.3) at resonance case, and establish some existence theorems under nonlinear growth restriction of $f$. The boundary value problems (1.1), (1.2) and (1.1), (1.3) with $n=2$ have been studied by [8]. Our results generalize the corresponding result in [8]. Our method is based upon the coincidence degree theory of Mawhin [13, 14]. Finally, we also give some examples to demonstrate our results.

Now, we will briefly recall some notations and an abstract existence result.

Let $Y, Z$ be real Banach spaces, let $L: \operatorname{dom} L \subset Y \rightarrow Z$ be a Fredholm map of index zero, and let $P: Y \rightarrow Y, Q: Z \rightarrow Z$ be continuous projectors such that $\operatorname{Im} P=\operatorname{Ker} L, \operatorname{Ker} Q=\operatorname{Im} L$, and $Y=\operatorname{Ker} L \oplus \operatorname{Ker} P, Z=\operatorname{Im} L \oplus \operatorname{Im} Q$. It follows that $\left.L\right|_{\operatorname{dom} L \cap \operatorname{Ker} P}: \operatorname{dom} L \cap \operatorname{Ker} P \rightarrow \operatorname{Im} L$ is invertible. We denote the inverse of that map by $K_{P}$. If $\Omega$ is an open-bounded subset of $Y$ such that dom $L \cap \Omega \neq \varnothing$, the map $N: Y \rightarrow Z$ will be called $L$-compact on $\bar{\Omega}$ if $Q N(\bar{\Omega})$ is bounded and $K_{P}(I-Q) N: \bar{\Omega} \rightarrow Y$ is compact.

The theorem we use is of [13, Theorem 2.4] or of [14, Theorem IV.13].

Theorem 1.1 (see[13,14]). Let $L$ be a Fredholm operator of index zero and let $N$ be L-compact on $\bar{\Omega}$. Assume that the following conditions are satisfied:

(i) $L x \neq \lambda N x$ for every $(x, \lambda) \in(\operatorname{dom} L \backslash \operatorname{Ker} L) \cap \partial \Omega \times(0,1)$;

(ii) $N x \notin \operatorname{Im} L$ for every $x \in \operatorname{Ker} L \cap \partial \Omega$;

(iii) $\operatorname{deg}\left(\left.Q N\right|_{\operatorname{Ker} L,}, \cap \operatorname{Ker} L, 0\right) \neq 0$, where $Q: Z \rightarrow Z$ is a projection as above with $\operatorname{Im} L=$ $\operatorname{Ker} Q$.

Then the equation $L x=N x$ has at least one solution in $\operatorname{dom} L \cap \bar{\Omega}$.

We use the classical space $C^{n-1}[0,1]$, for $x \in C^{n-1}[0,1]$, we use the norm $\|x\|=$ $\max \left\{\|x\|_{\infty},\left\|x^{\prime}\right\|_{\infty}, \ldots,\left\|x^{(n-1)}\right\|_{\infty}\right\}$, the norm $\left\|x^{(i)}\right\|_{\infty}=\max _{t \in[0,1]}\left|x^{(i)}(t)\right|, i=0,1, \ldots, n-1$, and denote the norm in $Z=L^{1}[0,1]$ by $\|\cdot\|_{1}$. We also use the Sobolev space

$$
\begin{aligned}
W^{n, 1}(0,1)= & \left\{x:[0,1] \longrightarrow \mathbb{R} \mid x, x^{\prime}, \ldots, x^{(n-1)}\right. \text { that are absolutely } \\
& \text { continuous on } \left.[0,1] \text { with } x^{(n)} \in L^{1}[0,1]\right\} .
\end{aligned}
$$

Throughout this paper, we assume that the $\beta_{j}$ 's have not the same sign, or there exist $j_{1}, j_{2} \in\{1,2, \ldots, m-2\}$ such that $\operatorname{sign}\left(\beta_{j_{1}} \cdot \beta_{j_{2}}\right)=-1$.

\section{Main results}

In this section, we will firstly prove existence results for BVP (1.1), (1.2). To do this, we let $Y=C^{n-1}[0,1], Z=L^{1}[0,1]$ and let $L$ be the linear operator $L: \operatorname{dom} L \subset Y \rightarrow Z$ with 


$$
\begin{aligned}
& \operatorname{dom} L=\left\{u \in W^{n, 1}(0,1): u^{(i)}(0)=0, i=1,2, \ldots, n-2,\right. \\
& \left.u^{(n-1)}(0)=u^{(n-1)}(\xi), \quad u^{(n-2)}(1)=\sum_{j=1}^{m-2} \beta_{j} u^{(n-2)}\left(\eta_{j}\right)\right\},
\end{aligned}
$$

and $L u=u^{(n)}, u \in \operatorname{dom} L$. We also define $N: Y \rightarrow Z$ by setting

$$
N u=f\left(t, u(t), \ldots, u^{(n-1)}(t)\right)+e(t), \quad t \in(0,1) .
$$

Then BVP (1.1), (1.2) can be written by $L u=N u$.

Lemma 2.1. If $\sum_{j=1}^{m-2} \beta_{j}=1, \sum_{j=1}^{m-2} \beta_{j} \eta_{j} \neq 1$, then $L: \operatorname{dom} L \subset Y \rightarrow Z$ is a Fredholm operator of index zero. Furthermore, the linear continuous projector operator $Q: Z \rightarrow Z$ can be defined by

$$
Q v=\frac{1}{\xi} \int_{0}^{\xi} v\left(s_{1}\right) d s_{1}
$$

and linear operator $K_{P}: \operatorname{Im} L \rightarrow \operatorname{dom} L \cap$ Ker $P$ can be written by

$$
K_{P} v=\frac{t^{n-1}}{(n-1) !\left(\sum_{j=1}^{m-2} \beta_{j} \eta_{j}-1\right)} \sum_{j=1}^{m-2} \beta_{j} \int_{\eta_{j}}^{1} \int_{0}^{s_{1}} v\left(s_{1}\right) d s_{1} d s_{2}+\int_{0}^{t} \int_{0}^{s_{n}} \cdots \int_{0}^{s_{2}} v\left(s_{1}\right) d s_{1} \cdots d s_{n}
$$

with

$$
\left\|K_{P} v\right\| \leq \Delta_{1}\|v\|_{1}, \quad \forall v \in \operatorname{Im} L
$$

where

$$
\Delta_{1}=1+\frac{1}{\left|\sum_{j=1}^{m-2} \beta_{j} \eta_{j}-1\right|} \sum_{j=1}^{m-2}\left|\beta_{j}\right|\left(1-\eta_{j}\right)
$$

Proof. It is clear that $\operatorname{Ker} L=\{u \in \operatorname{dom} L: u=d, d \in \mathbb{R}\}$. We now show that

$$
\operatorname{Im} L=\left\{v \in Z: \int_{0}^{\xi} v\left(s_{1}\right) d s_{1}=0\right\}
$$

Since the equation

$$
u^{(n)}=v
$$


has solution $u(t)$ which satisfies

$$
\begin{gathered}
u^{(i)}(0)=0, \quad i=1,2, \ldots, n-2, \\
u^{(n-1)}(0)=u^{(n-1)}(\xi) \\
u^{(n-2)}(1)=\sum_{j=1}^{m-2} \beta_{j} u^{(n-2)}\left(\eta_{j}\right)
\end{gathered}
$$

if and only if

$$
\int_{0}^{\xi} v\left(s_{1}\right) d s_{1}=0
$$

In fact, if (2.8) has solution $u(t)$ satisfying (2.9), then

$$
\int_{0}^{\xi} v\left(s_{1}\right) d s_{1}=\int_{0}^{\xi} u^{(n)}\left(s_{1}\right) d s_{1}=u^{(n-1)}(\xi)-u^{(n-1)}(0)=0 .
$$

On the other hand, if (2.10) holds, setting

$$
u(t)=c_{0}+c t^{n-1}+\int_{0}^{t} \int_{0}^{s_{n}} \cdots \int_{0}^{s_{2}} v\left(s_{1}\right) d s_{1} \cdots d s_{n}
$$

where $c_{0}$ is an arbitrary constant, $c=\sum_{j=1}^{m-2} \beta_{j} \int_{\eta_{j}}^{1} \int_{0}^{s_{2}} v\left(s_{1}\right) d s_{1} d s_{2} /(n-1) !\left(\sum_{j=1}^{m-2} \beta_{j} \eta_{j}-1\right)$, then $u(t)$ is a solution of (2.8), and satisfies (2.9). Hence (2.7) holds.

For $v \in Z$, taking the projector

$$
Q v=\frac{1}{\xi} \int_{0}^{\xi} v\left(s_{1}\right) d s_{1}
$$

Let $v_{1}=v-Q v$. By $\int_{0}^{\xi} v_{1}\left(s_{1}\right) d s_{1}=0$, then $v_{1} \in \operatorname{Im} L$, hence $Z=\operatorname{Im} L+\mathbb{R}$. Since $\operatorname{Im} L \cap \mathbb{R}=\{0\}$, we have $Z=\operatorname{Im} L \oplus \mathbb{R}$, thus

$$
\operatorname{dim} \operatorname{Ker} L=\operatorname{dim} \mathbb{R}=\operatorname{codim} \operatorname{Im} L=1 \text {. }
$$

Hence $L$ is a Fredholm operator of index zero.

Taking $P: Y \rightarrow Y$ as follows:

$$
P u=u(0),
$$

then the generalized inverse $K_{P}: \operatorname{Im} L \rightarrow \operatorname{dom} L \cap \operatorname{Ker} P$ of $L$ can be written by

$$
K_{P} v=\frac{t^{n-1}}{(n-1) !\left(\sum_{j=1}^{m-2} \beta_{j} \eta_{j}-1\right)} \sum_{j=1}^{m-2} \beta_{j} \int_{\eta_{j}}^{1} \int_{0}^{s_{2}} v\left(s_{1}\right) d s_{1} d s_{2}+\int_{0}^{t} \int_{0}^{s_{n}} \cdots \int_{0}^{s_{2}} v\left(s_{1}\right) d s_{1} \cdots d s_{n} .
$$


In fact, for $v \in \operatorname{Im} L$, we have

$$
\left(L K_{P}\right) v=\left(K_{P} v\right)^{(n)}(t)=v(t)
$$

and for all $u \in \operatorname{dom} L \cap \operatorname{Ker} P$, we have

$$
\begin{aligned}
\left(K_{P} L\right) u & =\frac{t^{n-1}}{(n-1) !\left(\sum_{j=1}^{m-2} \beta_{j} \eta_{j}-1\right)} \sum_{j=1}^{m-2} \beta_{j} \int_{\eta_{j}}^{1} \int_{0}^{s_{2}} u^{(n)}\left(s_{1}\right) d s_{1} d s_{2}+\int_{0}^{t} \int_{0}^{s_{n}} \cdots \int_{0}^{s_{2}} u^{(n)}\left(s_{1}\right) d s_{1} \cdots d s_{n} \\
& =u(t)-u(0) .
\end{aligned}
$$

In view of $u \in \operatorname{dom} L \cap \operatorname{Ker} P, P u=u(0)=0$, thus

$$
\left(K_{P} L\right) u(t)=u(t)
$$

this shows that $K_{P}=\left(\left.L\right|_{\operatorname{dom} L \cap \operatorname{Ker} P}\right)^{-1}$.

Again since for $i=0,1, \ldots, n-1$, we have

$$
\left(K_{P} v\right)^{(i)}(t)=\frac{t^{n-1-i}}{(n-1-i) !\left(\sum_{j=1}^{m-2} \beta_{j} \eta_{j}-1\right)} \sum_{j=1}^{m-2} \beta_{j} \int_{\eta_{j}}^{1} \int_{0}^{s_{2}} v\left(s_{1}\right) d s_{1} d s_{2}+\int_{0}^{t} \int_{0}^{s_{n-i}} \cdots \int_{0}^{s_{2}} v\left(s_{1}\right) d s_{1} \cdots d s_{n-i}
$$

consequently, for $i=0,1, \ldots, n-1$, we have

$$
\left|\left(K_{P} v\right)^{(i)}(t)\right| \leq\left[\frac{1}{\left|\sum_{j=1}^{m-2} \beta_{j} \eta_{j}-1\right|} \sum_{j=1}^{m-2}\left|\beta_{j}\right|\left(1-\eta_{j}\right)+1\right]\|v\|_{1}=\Delta_{1}\|v\|_{1}
$$

where $\Delta_{1}=\left(1 /\left|\sum_{j=1}^{m-2} \beta_{j} \eta_{j}-1\right|\right) \sum_{j=1}^{m-2}\left|\beta_{j}\right|\left(1-\eta_{j}\right)+1$. Thus

$$
\left\|\left(K_{P} v\right)^{(i)}\right\|_{\infty} \leq \Delta_{1}\|v\|_{1}, \quad i=0,1, \ldots, n-1,
$$

then $\left\|K_{P} v\right\| \leq \Delta_{1}\|v\|_{1}$. This completes the proof of Lemma 2.1.

Theorem 2.2. Let $f:[0,1] \times \mathbb{R}^{n} \rightarrow \mathbb{R}$ be a continuous function. Assume that there exists $n_{1} \in$ $\{1,2, \ldots, m-3\}(m \geq 4)$ such that $\beta_{j}>0\left(j=1,2, \ldots, n_{1}\right), \beta_{j}<0 \quad\left(j=n_{1}+1, n_{1}+2, \ldots, m-2\right)$. Furthermore, the following conditions are satisfied:

$\left(\mathrm{A}_{1}\right) \sum_{j=1}^{m-2} \beta_{j}=1, \sum_{j=1}^{m-2} \beta_{j} \eta_{j} \neq 1$;

$\left(\mathrm{A}_{2}\right)$ there exist functions $a_{0}, a_{1}, \ldots, a_{n-1}, b, r \in L^{1}[0,1]$, constant $\sigma \in[0,1)$, and some $j \in$ $\{0,1, \ldots, n-1\}$ such that for all $\left(u_{0}, u_{1}, \ldots, u_{n-1}\right) \in \mathbb{R}^{n}, t \in[0,1]$,

$$
\left|f\left(t, u_{0}, \ldots, u_{n-1}\right)\right| \leq \sum_{i=0}^{n-1} a_{i}(t)\left|u_{i}\right|+b(t)\left|u_{j}\right|^{\sigma}+r(t)
$$


$\left(\mathrm{A}_{3}\right)$ there exists $M>0$ such that for $\left(u_{1}, u_{2}, \ldots, u_{n-1}\right) \in \mathbb{R}^{n-1}$, if $|u|>M$, then

$$
\left|f\left(t, u, u_{1}, \ldots, u_{n-1}\right)\right| \geq \alpha|u|-\sum_{i=1}^{n-1} \alpha_{i}\left|u_{i}\right|-\gamma, \quad t \in[0,1]
$$

where $\alpha>0, \alpha_{i} \geq 0, i=1,2, \ldots, n-1, \gamma \geq 0$;

$\left(\mathrm{A}_{4}\right)$ there exists $M^{*}>0$ such that for any $d \in \mathbb{R}$, if $|d|>M^{*}$, then either

$$
d \cdot f(t, d, 0, \ldots, 0) \leq 0
$$

or

$$
d \cdot f(t, d, 0, \ldots, 0) \geq 0
$$

Then, for every $e \in L^{1}[0,1], B V P(1.1),(1.2)$ has at least one solution in $C^{n-1}[0,1]$ provided that $\sum_{i=0}^{n-1}\left\|a_{i}\right\|_{1}<1 / \Delta_{2}$, where $\Delta_{2}=\Delta_{1}+1+(1 / \alpha) \sum_{i=1}^{n-1} \alpha_{i}, \Delta_{1}$ as in Lemma 2.1 .

Proof. Set

$$
\Omega_{1}=\{u \in \operatorname{dom} L \backslash \operatorname{Ker} L: L u=\lambda N u, \lambda \in[0,1]\}
$$

Then for $u \in \Omega_{1}, L u=\lambda N u$, thus $\lambda \neq 0, N u \in \operatorname{Im} L=\operatorname{Ker} Q$. Hence

$$
\int_{0}^{\xi}\left[f\left(t, u(t), \ldots, u^{(n-1)}(t)\right)+e(t)\right] d t=0 .
$$

Thus, there exists $t_{0} \in[0, \xi]$ such that

$$
f\left(t_{0}, u\left(t_{0}\right), u^{\prime}\left(t_{0}\right), \ldots, u^{(n-1)}\left(t_{0}\right)\right)=-\frac{1}{\xi} \int_{0}^{\xi} e(t) d t .
$$

This yields

$$
\left|f\left(t_{0}, u\left(t_{0}\right), u^{\prime}\left(t_{0}\right), \ldots, u^{(n-1)}\left(t_{0}\right)\right)\right| \leq \frac{1}{\xi}\|e\|_{1} .
$$

If for some $t_{1} \in[0,1],\left|u\left(t_{1}\right)\right| \leq M$, then we have

$$
|u(0)|=\left|u\left(t_{1}\right)-\int_{0}^{t_{1}} u^{\prime}(t) d t\right| \leq M+\left\|u^{\prime}\right\|_{\infty} .
$$

Otherwise, if $|u(t)|>M$ for any $t \in[0,1]$, from (2.30) and $\left(\mathrm{A}_{3}\right)$, we obtain

$$
\begin{aligned}
\left|u\left(t_{0}\right)\right| & \leq \frac{1}{\alpha} \sum_{i=1}^{n-1} \alpha_{i}\left|u^{(i)}\left(t_{0}\right)\right|+\frac{1}{\alpha}\left(\gamma+\frac{1}{\xi}\|e\|_{1}\right) \\
& \leq \frac{1}{\alpha} \sum_{i=1}^{n-1} \alpha_{i}\left\|u^{(i)}\right\|_{\infty}+\frac{1}{\alpha}\left(\gamma+\frac{1}{\xi}\|e\|_{1}\right) .
\end{aligned}
$$


Thus

$$
\begin{aligned}
|u(0)| & =\left|u\left(t_{0}\right)-\int_{0}^{t_{0}} u^{\prime}(t) d t\right| \\
& \leq\left|u\left(t_{0}\right)\right|+\left\|u^{\prime}\right\|_{\infty} \\
& \leq \frac{1}{\alpha} \sum_{i=1}^{n-1} \alpha_{i}\left\|u^{(i)}\right\|_{\infty}+\frac{1}{\alpha}\left(\gamma+\frac{1}{\xi}\|e\|_{1}\right)+\left\|u^{\prime}\right\|_{\infty} .
\end{aligned}
$$

Again, since $u^{(i)}(0)=0, i=1,2, \ldots, n-2$, then for all $t \in[0,1]$, we have

$$
\left|u^{(i)}(t)\right|=\left|u^{(i)}(0)+\int_{0}^{t} u^{(i+1)}(t) d t\right| \leq\left\|u^{(i+1)}\right\|_{\infty} .
$$

Thus

$$
\left\|u^{(i)}\right\|_{\infty} \leq\left\|u^{(i+1)}\right\|_{\infty} \quad i=1,2, \ldots, n-2
$$

Therefore, we have

$$
\left\|u^{(i)}\right\|_{\infty} \leq\left\|u^{(n-1)}\right\|_{\infty}, \quad i=1,2, \ldots, n-2
$$

Hence

$$
\|P u\|=|u(0)| \leq\left(1+\frac{1}{\alpha} \sum_{i=1}^{n-1} \alpha_{i}\right)\left\|u^{(n-1)}\right\|_{\infty}+\frac{1}{\alpha}\left(\gamma+\frac{1}{\xi}\|e\|_{1}\right)+M
$$

According to the conditions $\beta_{j}>0\left(j=1,2, \ldots, n_{1}\right), \beta_{j}<0\left(j=n_{1}+1, n_{1}+2, \ldots, m-2\right)$, and $u^{(n-2)}(1)=\sum_{j=1}^{m-2} \beta_{j} u^{(n-2)}\left(\eta_{j}\right)$, we have

$$
u^{(n-2)}(1)-\sum_{j=n_{1}+1}^{m-2} \beta_{j} u^{(n-2)}\left(\eta_{j}\right)=\sum_{j=1}^{n_{1}} \beta_{j} u^{(n-2)}\left(\eta_{j}\right)
$$

Again, since there exist $t_{2} \in\left[\eta_{n_{1}+1}, 1\right], t_{3} \in\left[\eta_{1}, \eta_{n_{1}}\right]$ such that

$$
\begin{gathered}
u^{(n-2)}\left(t_{2}\right)=\frac{1}{1-\sum_{j=n_{1}+1}^{m-2} \beta_{j}}\left[u^{(n-2)}(1)-\sum_{j=n_{1}+1}^{m-2} \beta_{j} u^{(n-2)}\left(\eta_{j}\right)\right], \\
u^{(n-2)}\left(t_{3}\right)=\frac{1}{1-\sum_{j=1}^{n_{1}} \beta_{j}} \sum_{j=1}^{n_{1}} \beta_{j} u^{(n-2)}\left(\eta_{j}\right)
\end{gathered}
$$

thus, in view of $\sum_{j=1}^{m-2} \beta_{j}=1$, from (2.38)-(2.40), we get

$$
u^{(n-2)}\left(t_{2}\right)=u^{(n-2)}\left(t_{3}\right)
$$


Since $\eta_{n_{1}}<\eta_{n_{1}+1}$, then $t_{2} \neq t_{3}$, so from $(2.41)$, there exists $t^{*} \in\left(t_{2}, t_{3}\right)$ such that $u^{(n-1)}\left(t^{*}\right)=0$. Hence, in view of $u^{(n-1)}(t)=u^{(n-1)}\left(t^{*}\right)+\int_{t^{*}}^{t} u^{(n)}(t) d t$, we have

$$
\left\|u^{(n-1)}\right\|_{\infty} \leq\left\|u^{(n)}\right\|_{1}=\|L u\|_{1} \leq\|N u\|_{1} .
$$

Therefore, from (2.37) and (2.42), one has

$$
\|P u\| \leq\left(1+\frac{1}{\alpha} \sum_{i=1}^{n-1} \alpha_{i}\right)\|N u\|_{1}+\frac{1}{\alpha}\left(\gamma+\frac{1}{\xi}\|e\|_{1}\right)+M
$$

Again, for $u \in \Omega_{1}, u \in \operatorname{dom} L \backslash \operatorname{Ker} L$, then $(I-P) u \in \operatorname{dom} L \backslash \operatorname{Ker} L, L P u=0$. Thus from Lemma 2.1, we have

$$
\begin{aligned}
\|(I-P) u\| & =\left\|K_{p} L(I-P) u\right\| \\
& \leq \Delta_{1}\|L(I-P) u\|_{1} \\
& =\Delta_{1}\|L u\|_{1} \\
& \leq \Delta_{1}\|N u\|_{1} .
\end{aligned}
$$

From (2.43) and (2.44), we get

$$
\begin{aligned}
\|u\| & \leq\|P u\|+\|(I-P) u\| \\
& \leq\left(1+\Delta_{1}+\frac{1}{\alpha} \sum_{i=1}^{n-1} \alpha_{i}\right)\|N u\|_{1}+c_{1} \\
& =\Delta_{2}\|N u\|_{1}+c_{1}
\end{aligned}
$$

where $c_{1}=M+(1 / \alpha)\left(\gamma+(1 / \xi)\|e\|_{1}\right)$.

If $\left(2.23_{j}\right)_{n-1}$ holds, then from (2.45), we get

$$
\|u\| \leq \Delta_{2}\left[\sum_{i=0}^{n-1}\left\|a_{i}\right\|_{1}\left\|u^{(i)}\right\|_{\infty}+\|b\|_{1}\left\|u^{(n-1)}\right\|_{\infty}^{\sigma}+c\right]
$$

where $c=\|r\|_{1}+\|e\|_{1}+c_{1} / \Delta_{2}$. In view of (2.46), we obtain

$$
\|u\|_{\infty} \leq\|u\| \leq \frac{\Delta_{2}}{1-\Delta_{2}\left\|a_{0}\right\|_{1}}\left[\sum_{i=1}^{n-1}\left\|a_{i}\right\|_{1}\left\|u^{(i)}\right\|_{\infty}+\|b\|_{1}\left\|u^{(n-1)}\right\|_{\infty}^{\sigma}+c\right] .
$$

Again, $\left\|u^{\prime}\right\|_{\infty} \leq\|u\|$, from (2.46) and (2.47), one has

$$
\left\|u^{\prime}\right\|_{\infty} \leq \frac{\Delta_{2}}{1-\Delta_{2}\left(\left\|a_{0}\right\|_{1}+\left\|a_{1}\right\|_{1}\right)}\left[\sum_{i=2}^{n-1}\left\|a_{i}\right\|_{1}\left\|u^{(i)}\right\|_{\infty}+\|b\|_{1}\left\|u^{(n-1)}\right\|_{\infty}^{\sigma}+c\right] .
$$


In general, for $k=2,3, \ldots, n-2$, we have

$$
\begin{gathered}
\left\|u^{(k)}\right\|_{\infty} \leq \frac{\Delta_{2}}{1-\Delta_{2} \sum_{i=0}^{k}\left\|a_{i}\right\|_{1}}\left[\sum_{i=k+1}^{n-1}\left\|a_{i}\right\|_{1}\left\|u^{(i)}\right\|_{\infty}+\|b\|_{1}\left\|u^{(n-1)}\right\|_{\infty}^{\sigma}+c\right], \\
\left\|u^{(n-1)}\right\|_{\infty} \leq \frac{\Delta_{2}\|b\|_{1}}{1-\Delta_{2} \sum_{i=0}^{n-1}\left\|a_{i}\right\|_{1}}\left\|u^{(n-1)}\right\|_{\infty}^{\sigma}+\frac{\Delta_{2} c}{1-\Delta_{2} \sum_{i=0}^{n-1}\left\|a_{i}\right\|_{1}} .
\end{gathered}
$$

Since $\sigma \in[0,1)$, then from $(2.50)$, there exists $M_{n-1}>0$ such that $\left\|u^{(n-1)}\right\|_{\infty} \leq M_{n-1}$. Thus from $\left(2.49_{k}\right)$, there exist $M_{k}>0, k=0,1, \ldots, n-2$, such that $\left\|u^{(k)}\right\|_{\infty} \leq M_{k}, k=0,1, \ldots, n-2$. Hence

$$
\begin{aligned}
\|u\| & =\max \left\{\|u\|_{\infty},\left\|u^{\prime}\right\|_{\infty}, \ldots,\left\|u^{(n-1)}\right\|_{\infty}\right\} \\
& \leq \max \left\{M_{0}, M_{1}, \ldots, M_{n-1}\right\} .
\end{aligned}
$$

Therefore, $\Omega_{1}$ is bounded.

If $\left(2.23_{j}\right), j \in\{0,1, \ldots, n-2\}$ holds, similar to $\left(2.23_{j}\right)_{n-1}$ argument, we can prove that $\Omega_{1}$ is bounded too.

Set

$$
\Omega_{2}=\{u \in \operatorname{Ker} L: N u \in \operatorname{Im} L\}
$$

Then for $u \in \Omega_{2}, u \in \operatorname{Ker} L=\{u \in \operatorname{dom} L: u=d, d \in \mathbb{R}\}$, and $Q N u=0$, one has

$$
\int_{0}^{\xi}[f(t, d, 0, \ldots, 0)+e(t)] d t=0
$$

Thus, there exists $t_{4} \in[0, \xi]$ such that

$$
f\left(t_{4}, d, 0, \ldots, 0\right)=-\frac{1}{\xi} \int_{0}^{\xi} e(t) d t .
$$

This yields

$$
\left|f\left(t_{4}, d, 0, \ldots, 0\right)\right| \leq \frac{1}{\xi}\|e\|_{1} .
$$

Since either $|d| \leq M$ or $|d|>M$, if $|d|>M$, then in view of $\left(\mathrm{A}_{3}\right)$ and (2.55), we have $|d| \leq$ $(1 / \alpha)\left(\gamma+(1 / \xi)\|e\|_{1}\right)$. Therefore, it follows that

$$
|d| \leq \max \left\{M, \frac{1}{\alpha}\left(r+\frac{1}{\xi}\|e\|_{1}\right)\right\}
$$

Hence $\Omega_{2}$ is bounded. 
Now, according to condition $\left(\mathrm{A}_{4}\right)$, we have the following two cases.

Case 1. For any $d \in \mathbb{R}$, if $|d|>M^{*}$, then $d \cdot f(t, d, 0, \ldots, 0) \leq 0, t \in[0,1]$. In this case, we set

$$
\Omega_{3}=\{u \in \operatorname{Ker} L:-(1-\lambda) J u+\lambda Q N u=0, \lambda \in[0,1]\},
$$

where $J: \operatorname{Ker} L \rightarrow \operatorname{Im} Q$ is the linear isomorphism given by $J(d)=d, d \in \mathbb{R}$.

In the following, we will show that $\Omega_{3}$ is bounded. Suppose $u_{n}(t)=d_{n} \in \Omega_{3}$ and $\left|d_{n}\right| \rightarrow$ $\infty(n \rightarrow \infty)$, then there exists $\lambda_{n} \in[0,1]$, for sufficiently large $n$, such that

$$
1-\lambda_{n}=\lambda_{n} \cdot \frac{Q N\left(d_{n}\right)}{d_{n}} .
$$

Since $\lambda_{n} \in[0,1]$, then $\left\{\lambda_{n}\right\}$ has a convergent subsequence, and we write (for simplicity of notation) $\lambda_{n} \rightarrow \lambda_{0}(n \rightarrow \infty)$.

If $\left(2.23_{j}\right)_{j}, j \in\{1,2, \ldots, n-1\}$ holds, then

$$
\begin{aligned}
\left|\frac{Q N\left(d_{n}\right)}{d_{n}}\right| & =\frac{1}{\left|d_{n}\right|}\left|\frac{1}{\xi} \int_{0}^{\xi}\left[f\left(t, d_{n}, 0, \ldots, 0\right)+e(t)\right] d t\right| \\
& \leq \frac{1}{\left|d_{n}\right|} \frac{1}{\xi}\left(\left\|a_{0}\right\|_{1}\left|d_{n}\right|+\|r\|_{1}+\|e\|_{1}\right) \\
& =\frac{1}{\xi}\left\|a_{0}\right\|_{1}+\frac{1}{\xi} \frac{\|r\|_{1}+\|e\|_{1}}{\left|d_{n}\right|} .
\end{aligned}
$$

If $\left(2.23_{j}\right)_{0}$ holds, then

$$
\begin{aligned}
\left|\frac{Q N\left(d_{n}\right)}{d_{n}}\right| & =\frac{1}{\left|d_{n}\right|}\left|\frac{1}{\xi} \int_{0}^{\xi}\left[f\left(t, d_{n}, 0, \ldots, 0\right)+e(t)\right] d t\right| \\
& \leq \frac{1}{\left|d_{n}\right|} \frac{1}{\xi}\left(\left\|a_{0}\right\|_{1}\left|d_{n}\right|+\|b\|_{1}\left|d_{n}\right|^{\sigma}+\|r\|_{1}+\|e\|_{1}\right) \\
& =\frac{1}{\xi}\left\|a_{0}\right\|_{1}+\frac{1}{\xi} \cdot \frac{\|b\|_{1}}{\left|d_{n}\right|^{1-\sigma}}+\frac{1}{\xi} \cdot \frac{\|r\|_{1}+\|e\|_{1}}{\left|d_{n}\right|} .
\end{aligned}
$$

Since $\left|d_{n}\right| \rightarrow \infty$, then from (2.59) or (2.60), we know $\left\{\left|Q N\left(d_{n}\right) / d_{n}\right|\right\}$ is bounded. From (2.58), we have $\lambda_{n} \rightarrow \lambda_{0} \neq 0$. Hence for $n$ sufficiently large, $\lambda_{n} \neq 0$, and we have

$$
\frac{1-\lambda_{n}}{\lambda_{n}}=\frac{1}{\xi}\left[\int_{0}^{\xi} \frac{f\left(t, d_{n}, 0, \ldots, 0\right)}{d_{n}} d t+\frac{1}{d_{n}} \int_{0}^{\xi} e(t) d t\right] .
$$

In view of $\left|d_{n}\right| \rightarrow \infty$, we can assume that $\left|d_{n}\right|>\max \left\{M, M^{*}\right\}$, thus for $n$ sufficiently large, from $\left(\mathrm{A}_{3}\right)$, we get

$$
\left|\frac{f\left(t, d_{n}, 0, \ldots, 0\right)}{d_{n}}\right| \geq \alpha-\frac{\gamma}{\left|d_{n}\right|} \geq \frac{\alpha}{2}>0 .
$$

Again since $d_{n} \cdot f\left(t, d_{n}, 0, \ldots, 0\right) \leq 0, t \in[0,1]$, from (2.62), one has

$$
\frac{f\left(t, d_{n}, 0, \ldots, 0\right)}{d_{n}} \leq-\frac{\alpha}{2}<0 .
$$


Hence, according to Fatou lemma, we obtain

$$
\begin{aligned}
\varlimsup_{n \rightarrow \infty}\left[\int_{0}^{\xi} \frac{f\left(t, d_{n}, 0, \ldots, 0\right)}{d_{n}} d t+\frac{1}{d_{n}} \int_{0}^{\xi} e(t) d t\right] & \leq \varlimsup_{n \rightarrow \infty} \int_{0}^{\xi} \frac{f\left(t, d_{n}, 0, \ldots, 0\right)}{d_{n}} d t \\
& \leq \int_{0}^{\xi} \varlimsup_{n \rightarrow \infty} \frac{f\left(t, d_{n}, 0, \ldots, 0\right)}{d_{n}} d t \\
& \leq-\frac{\alpha}{2} \xi<0,
\end{aligned}
$$

which contradicts with $\left(1-\lambda_{n}\right) / \lambda_{n} \geq 0$. Thus $\Omega_{3}$ is bounded.

Case 2. For any $d \in \mathbb{R}$, if $|d|>M^{*}$, then $d \cdot f(t, d, 0, \ldots, 0) \geq 0, t \in[0,1]$. In this case, we set

$$
\Omega_{3}=\{u \in \operatorname{Ker} L:(1-\lambda) J u+\lambda Q N u=0, \lambda \in[0,1]\},
$$

where $J$ as in above. Similar to the above argument, we can also show that $\Omega_{3}$ is bounded.

In the following, we will prove that all the conditions of Theorem 1.1 are satisfied. Set $\Omega$ to be an open-bounded subset of $Y$ such that $\bigcup_{i=1}^{3} \bar{\Omega}_{i} \subset \Omega$. By using the Ascoli-Arzela theorem, we can prove that $K_{P}(I-Q) N: Y \rightarrow Y$ is compact, thus $N$ is $L$-compact on $\bar{\Omega}$. Then by the above argument, we have the following.

(i) $L u \neq \lambda N u$ for every $(u, \lambda) \in[(\operatorname{dom} L \backslash \operatorname{Ker} L) \cap \partial \Omega] \times(0,1)$.

(ii) $N u \notin \operatorname{Im} L$ for $u \in \operatorname{Ker} L \cap \partial \Omega$.

(ii) $H(u, \lambda)= \pm \lambda J u+(1-\lambda) Q N u$. According to the above argument, we know $H(u, \lambda) \neq 0$ for every $u \in \operatorname{Ker} L \cap \partial \Omega$. Thus, by the homotopy property of degree,

$$
\begin{aligned}
\operatorname{deg}\left(\left.Q N\right|_{\operatorname{Ker} L}, \Omega \cap \operatorname{Ker} L, 0\right) & =\operatorname{deg}(H(\cdot, 0), \Omega \cap \operatorname{Ker} L, 0) \\
& =\operatorname{deg}(H(\cdot, 1), \Omega \cap \operatorname{Ker} L, 0) \\
& =\operatorname{deg}( \pm J, \Omega \cap \operatorname{Ker} L, 0) \neq 0 .
\end{aligned}
$$

Then by Theorem 1.1, $L u=N u$ has at least one solution in $\operatorname{dom} L \cap \bar{\Omega}$, so that BVP (1.1), (1.2) has solution in $C^{n-1}[0,1]$. The proof is finished.

Now, we will consider existence results for BVP (1.1), (1.3). In the following, the mapping $N$ and linear operator $L$ are the same as above, and let

$$
\operatorname{dom} L=\left\{u \in W^{n, 1}(0,1): u^{(i)}(0)=0, i=1,2, \ldots, n-1, u^{(n-2)}(1)=\sum_{j=1}^{m-2} \beta_{j} u^{(n-2)}\left(\eta_{j}\right)\right\} .
$$

Lemma 2.3. If $\sum_{j=1}^{m-2} \beta_{j}=1, \sum_{j=1}^{m-2} \beta_{j} \eta_{j}^{2} \neq 1$, then $L: \operatorname{dom} L \subset Y \rightarrow Z$ is a Fredholm operator of index zero. Furthermore, the linear continuous projector $Q: Z \rightarrow Z$ can be defined by

$$
Q v=\frac{2}{1-\sum_{j=1}^{m-2} \beta_{j} \eta_{j}^{2}} \sum_{j=1}^{m-2} \beta_{j} \int_{\eta_{j}}^{1} \int_{0}^{s_{2}} v\left(s_{1}\right) d s_{1} d s_{2}
$$


and linear operator $K_{P}=\operatorname{Im} L \rightarrow \operatorname{dom} L \cap \operatorname{Ker} P$ can be written as

$$
K_{P} v=\int_{0}^{t} \int_{0}^{s_{n}} \cdots \int_{0}^{s_{2}} v\left(s_{1}\right) d s_{1} \cdots d s_{n}
$$

with

$$
\left\|K_{P} v\right\| \leq\|v\|_{1}, \quad \forall v \in \operatorname{Im} L
$$

Notice that the $\operatorname{Ker} L=\{u \in \operatorname{dom} L: u=d, d \in \mathbb{R}, t \in[0,1]\}$ and $\operatorname{Im} L=\{v \in Z$ : $\left.\sum_{j=1}^{m-2} \beta_{j} \int_{\eta_{j}}^{1} \int_{0}^{s_{2}} v\left(s_{1}\right) d s_{1} d s_{2}=0\right\}$. Thus, by using the same method as the proof of Lemma 2.1, we can prove Lemma 2.3 , and we omit it.

Theorem 2.4. Let $f:[0,1] \times \mathbb{R}^{n} \rightarrow \mathbb{R}$ be a continuous function. Assume that condition $\left(A_{2}\right)$ of Theorem 2.2 and the following conditions are satisfied:

$\left(\mathrm{A}_{5}\right) \sum_{j=1}^{m-2} \beta_{j}=1, \sum_{j=1}^{m-2} \beta_{j} \eta_{j}^{2} \neq 1$;

$\left(\mathrm{A}_{6}\right)$ there exists $M>0$, such that for $u \in \operatorname{dom} L$, if $|u(t)|>M$ for all $t \in[0,1]$, then

$$
\sum_{j=1}^{m-2} \beta_{j} \int_{\eta_{j}}^{1} \int_{0}^{s_{2}}\left[f\left(s_{1}, u\left(s_{1}\right), \ldots, u^{(n-1)}\left(s_{1}\right)\right)+e\left(s_{1}\right)\right] d s_{1} d s_{2} \neq 0
$$

( $\left.\mathrm{A}_{7}\right)$ there exists $M^{*}>0$ such that for any $d \in \mathbb{R}$, if $|d|>M^{*}$, then either

$$
d \cdot \sum_{j=1}^{m-2} \beta_{j} \int_{\eta_{j}}^{1} \int_{0}^{s_{2}}\left[f\left(s_{1}, d, 0, \ldots, 0\right)+e\left(s_{1}\right)\right] d s_{1} d s_{2}<0,
$$

or else

$$
d \cdot \sum_{j=1}^{m-2} \beta_{j} \int_{\eta_{j}}^{1} \int_{0}^{s_{2}}\left[f\left(s_{1}, d, 0, \ldots, 0\right)+e\left(s_{1}\right)\right] d s_{1} d s_{2}>0
$$

Then, for every $e \in L^{1}[0,1], B V P(1.1),(1.3)$ has at least one solution in $C^{n-1}[0,1]$ provided that $\sum_{i=0}^{n-1}\left\|a_{i}\right\|_{1}<1 / 2$.

The proof of Theorem 2.4 is similar to the proof of Theorem 2.2, and we omit it.

Next we give two examples to demonstrate the applications of the main results.

Example 2.5. Consider the boundary value problems

$$
\begin{gathered}
u^{\prime \prime \prime}(t)=f\left(t, u(t), u^{\prime}(t), u^{\prime \prime}(t)\right)+e(t), \quad t \in(0,1) \\
u^{\prime \prime}(0)=u^{\prime \prime}(\xi), \quad u^{\prime}(0)=0, \quad u^{\prime}(1)=6 u^{\prime}\left(\frac{1}{6}\right)-3 u^{\prime}\left(\frac{1}{3}\right)-2 u^{\prime}\left(\frac{1}{2}\right)
\end{gathered}
$$

where $f(t, u, v, w)=(1 / 22) u+(1 / 44) v+(1 / 44) w+\sin (w)^{1 / 5}, e \in L^{1}[0,1], \xi \in(0,1)$. 
Since $\beta_{1}=6, \beta_{2}=-3, \beta_{3}=-2, \eta_{1}=1 / 6, \eta_{2}=1 / 3, \eta_{3}=1 / 2$, then

(i) $\beta_{1}+\beta_{2}+\beta_{3}=1, \beta_{1} \eta_{1}+\beta_{2} \eta_{2}+\beta_{3} \eta_{3} \neq 1$;

(ii) $|f(t, u, v, w)| \leq(1 / 22)|u|+(1 / 44)|v|+(1 / 44)|w|+|w|^{1 / 5}$;

(iii) $|f(t, u, v, w)| \geq(1 / 22)|u|-(1 / 44)|v|-(1 / 44)|w|-1$;

(iv) for any $d \in \mathbb{R}, d \cdot f(t, d, 0,0)=(1 / 22) d^{2} \geq 0$.

Furthermore

$$
\begin{gathered}
\Delta_{1}=1+\frac{1}{\left|\sum_{j=1}^{3} \beta_{j} \eta_{j}-1\right|} \sum_{j=1}^{3}\left|\beta_{j}\right|\left(1-\eta_{j}\right)=5, \\
\Delta_{2}=\Delta_{1}+1+\frac{1}{\alpha} \sum_{i=1}^{2} \alpha_{i}=5+1+1=7, \\
\left\|a_{0}\right\|_{1}+\left\|a_{1}\right\|_{1}+\left\|a_{2}\right\|_{1}=\frac{1}{22}+\frac{1}{44}+\frac{1}{44}=\frac{1}{11}<\frac{1}{7} .
\end{gathered}
$$

Hence from Theorem 2.2, for every $e \in L^{1}[0,1]$, BVP (2.74) has at least one solution $u \in C^{2}[0,1]$. Example 2.6. Consider the boundary value problems

$$
\begin{gathered}
u^{\prime \prime \prime}(t)=f\left(t, u(t), u^{\prime}(t), u^{\prime \prime}(t)\right)+e(t), \quad t \in(0,1) \\
u^{\prime}(0)=0, \quad u^{\prime \prime}(0)=0, \quad u^{\prime}(1)=-4 u^{\prime}\left(\frac{1}{4}\right)+3 u^{\prime}\left(\frac{1}{3}\right)+2 u^{\prime}\left(\frac{1}{2}\right)
\end{gathered}
$$

where $f(t, u, v, w)=(1 / 8) u+(1 / 8) v+(1 / 8) w+\sin ^{2}(u) \sin (w)^{1 / 3}, e \in L^{1}[0,1], \xi \in(0,1)$.

Since $\beta_{1}=-4, \beta_{2}=3, \beta_{3}=2, \eta_{1}=1 / 4, \eta_{2}=1 / 3, \eta_{3}=1 / 2$, then

(i) $\beta_{1}+\beta_{2}+\beta_{3}=1, \beta_{1} \eta_{1}^{2}+\beta_{2} \eta_{2}^{2}+\beta_{3} \eta_{3}^{2} \neq 1$;

(ii) $|f(t, u, v, w)| \leq(1 / 8)|u|+(1 / 8)|v|+(1 / 8)|w|+|w|^{1 / 3}$;

(iii) let $M=8$, then for $|u(t)|>M$, one has

$$
\sum_{j=1}^{3} \beta_{j} \int_{\eta_{j}}^{1} \int_{0}^{s_{2}}\left[f\left(s_{1}, u\left(s_{1}\right), u^{\prime}\left(s_{1}\right), u^{\prime \prime}\left(s_{1}\right)\right)+e\left(s_{1}\right)\right] d s_{1} d s_{2} \neq 0
$$

(iv) for any $d \in \mathbb{R}$, one has

$$
d \sum_{j=1}^{3} \beta_{j} \int_{\eta_{j}}^{1} \int_{0}^{s_{2}} f\left(s_{1}, d, 0,0\right) d s_{1} d s_{2}=\frac{5}{192} d^{2}>0
$$

Furthermore

$$
\left\|a_{0}\right\|_{1}+\left\|a_{1}\right\|_{1}+\left\|a_{2}\right\|_{1}=\frac{1}{8}+\frac{1}{8}+\frac{1}{8}=\frac{3}{8}<\frac{1}{2}
$$

Hence from Theorem 2.4, for every $e \in L^{1}[0,1]$, BVP (2.76) has at least one solution $u \in C^{2}[0,1]$. 


\section{Acknowledgment}

The authors thank the referee for valuable suggestions which led to improvement of the original manuscript.

\section{References}

[1] Z. Du, W. Ge, and X. Lin, "Existence of solutions for a class of third-order nonlinear boundary value problems," Journal of Mathematical Analysis and Applications, vol. 294, no. 1, pp. 104-112, 2004.

[2] Z. Du, X. Lin, and W. Ge, "On a third-order multi-point boundary value problem at resonance," Journal of Mathematical Analysis and Applications, vol. 302, no. 1, pp. 217-229, 2005.

[3] W. Feng and J. R. L. Webb, "Solvability of $m$-point boundary value problems with nonlinear growth," Journal of Mathematical Analysis and Applications, vol. 212, no. 2, pp. 467-480, 1997.

[4] C. P. Gupta, "A generalized multi-point boundary value problem for second order ordinary differential equations," Applied Mathematics and Computation, vol. 89, no. 1-3, pp. 133-146, 1998.

[5] C. P. Gupta, S. K. Ntouyas, and P. Ch. Tsamatos, "On an $m$-point boundary-value problem for secondorder ordinary differential equations," Nonlinear Analysis: Theory, Methods \& Applications, vol. 23, no. 11, pp. 1427-1436, 1994.

[6] C. P. Gupta, S. K. Ntouyas, and P. Ch. Tsamatos, "Solvability of an $m$-point boundary value problem for second order ordinary differential equations," Journal of Mathematical Analysis and Applications, vol. 189, no. 2, pp. 575-584, 1995.

[7] B. Liu and J. Yu, "Solvability of multi-point boundary value problem at resonance. III," Applied Mathematics and Computation, vol. 129, no. 1, pp. 119-143, 2002.

[8] B. Liu, "Solvability of multi-point boundary value problem at resonance. II," Applied Mathematics and Computation, vol. 136, no. 2-3, pp. 353-377, 2003.

[9] R. Ma and N. Castaneda, "Existence of solutions of nonlinear $m$-point boundary-value problems," Journal of Mathematical Analysis and Applications, vol. 256, no. 2, pp. 556-567, 2001.

[10] R. K. Nagle and K. L. Pothoven, "On a third order nonlinear boundary value problem at resonance," Journal of Mathematical Analysis and Applications, vol. 195, no. 1, pp. 148-159, 1995.

[11] S. Lu and W. Ge, "On the existence of $m$-point boundary value problem at resonance for higher order differential equation," Journal of Mathematical Analysis and Applications, vol. 287, no. 2, pp. 522-539, 2003.

[12] P. K. Palamides, "Multi point boundary-value problems at resonance for $n$-order differential equations: positive and monotone solutions," Electronic Journal of Differential Equations, vol. 2004, no. 25, pp. 1-14, 2004.

[13] J. Mawhin, "Topological degree and boundary value problems for nonlinear differential equations," in Topological Methods for Ordinary Differential Equations, P. M. Fitzpertrick, M. Martelli, J. Mawhin, and R. Nussbaum, Eds., vol. 1537 of Lecture Notes in Mathematics, Springer, New York, NY, USA, 1991.

[14] J. Mawhin, Topological Degree Methods in Nonlinear Boundary Value Problems, vol. 40 of CBMS Regional Conference Series in Mathematics, American Mathematical Society, Providence, RI, USA, 1979. 